\title{
A Simple Optimization Model for Wireless Opportunistic Routing with Intra-session Network Coding
}

\author{
Fabio Soldo, Athina Markopoulou \\ University of California, Irvine \\ $\{$ fsoldo, athina $\} @ u c i . e d u$
}

\author{
Alberto Lopez Toledo \\ Telefonica Research \\ alopezt@tid.es
}

\begin{abstract}
In this paper, we consider the optimization of multipath opportunistic routing and congestion control in wireless mesh networks with intra-session network coding. We model this as a network utility maximization problem and we design a simple distributed solution that can be obtained by solving the dual problem. This formulation provides useful insight into the complex cross-layer interaction between intra-session network coding, multi-path routing and transport layer and a mathematical foundation for protocol design in this scenario.
\end{abstract}

\section{INTRODUCTION}

In this paper, we study the optimization of multi-path opportunistic routing and congestion control in wireless mesh networks with intra-session network coding. Opportunistic routing adapts to the varying dynamics of the wireless channel. At each transmission, different nodes may receive the transmitted packet due to the unpredictable losses. Moreover, if a node closer to the destination than the selected next hop experiences a successful reception, it will discard the received packet, as it is not the intended receipt. To overcome these limitations, opportunistic routing schemes do not chose the next hop node prior to the transmission. Instead, nodes that successfully received the packet (implicitly) coordinate to forward it towards the destination. By opportunistically exploiting successful reception of nodes closer to the destination, opportunistic routing significantly reduces the number of transmissions required to forward a packet to the destination [1], [2]. However, it also requires nodes to coordinate on which packets to transmit in order to avoid redundant transmission. As shown in [1], this problem is greatly simplified with the use of network coding.

Network coding enables nodes to code together buffered packets belonging to the same flow (intra-flow coding) or different flows (inter-flow coding). A node does not necessarily forward packets as they are generated by the source, but in general, it transmits a combination of them. This simplifies the scheduling since different nodes do not need to exchange information about which packets they transmit. They just need to ensure they will transmit innovative information.

Another benefit of network coding for wireless networks is its capability to mask random losses due to the varying nature of the wireless channel. This has been successfully exploited in recent work [3], where the authors illustrated a new network coding protocol specifically tailored to improve the performance of TCP over wireless mesh networks. Differently from prior approaches, [3] does not partition source packets into generations [4]. Instead, it defines a sliding window of packets to determine which packets should be coded together. Moreover, a certain amount of redundant packets are injected in the network to proactively account for the channel losses.

Although [3] describes in detail the network coding layer and its interaction with the TCP layer, it is not clear how they both interact with the routing layer. In this work, we focus on the joint optimization of opportunistic routing, congestion control and (intra-session) network coding in wireless mesh networks.

The work that is most related to ours is [5]. We consider the problem as in [5], but both our model and the decomposition method are different. In particular, we define different control variables that are not edge-specific but node-specific a metric that is more natural in wireless networks. This leads to a different primal model that is solved using a sub-gradient method applied to the dual problem. The main contributions of our paper are the simple model and the distributed solution that helps unveil the complex cross-layer interactions and provides further insights into the design of practical protocols. We formally derive a distributed solution to solve the optimization problem that highlights the necessity of two types of feedbacks: (i) end-to-end feedback to adjust the sending rate of the source based on the packet losses; and (ii) hopby-hop feedback to guarantee that the amount of information stored at the forwarding node has been relayed to the union of downstream nodes.

The rest of paper is organized as follows. In Section II we describe the scenario and notations used. In Section III, we formalize the model and we describe how to obtain a distributed solution. In Section IV, we discuss the insights gained into protocol design. In Section V, we position this work within the related literature. In Section VI, we conclude.

\section{ScEnARio And Notations}

Let us consider a wireless mesh network with $N$ nodes, modeled as a connectivity graph, $G(V, E)$, where $V$ is the set of nodes, such that $|V|=N$, and $E$ the set of logical pointto-point links. We assume that the topology is static. The time 
scale considered is such that we can assume a fixed available capacity $c_{i j},(i, j) \in E$ for every link (although, in general, this may vary a over longer time scales).

We consider a set of $K$ source-destination node pairs, $\left(s^{k}, d^{k}\right) \in S \times D$, where $k \in K$ and $S, D \subseteq V$, that want to exchange a certain amount of information. We assume that intermediate nodes use network coding with an opportunistic routing scheme (e.g. MORE [1]). In other words, before transmitting a packet a node performs a network coding operation on packets belonging to the same stream (intraflow network coding) and transmits the resulting coded packet. Moreover, the destination node of that transmission is not set prior to transmission. Instead, nodes that successfully receive the encoded packet implicitly coordinate to select the node in charge of transmitting the received packet downstream. This can be implemented using, for instance, the ETX metric [1], [2].

We denote with $z_{i}^{k}$ the number of packets sent by node $i$ belonging to the information flow $\left(s^{k}, d^{k}\right)$. We use $z_{i}$ to indicate the total number of packets sent from node $i$, $z_{i}=\sum_{k \in K} z_{i}^{k}$. We denote with $\sum_{j>i} z_{j}^{k}$ the sum of packets transmitted by nodes $j$ such that $(j, i) \in E$ and node $i$ is "closer" to $d^{k}$ than node $j$, according to the opportunistic routing metric used, that is, $i$ has a smaller ETX metric than $j$. This implies that $i$ is a potential forwarder of packets sent from node $j$. Similarly, $\sum_{j<i} z_{j}^{k}$ indicates the sum of packets transmitted by nodes $j$ such that $j$ is closer to $d^{k}$ than node $i$ (thus the notation " $j>i ")$.

Finally, we assume that the network coding operations are performed over a large enough Galois field to guarantee that a set of $n$ packets, associated with coding vectors embedded in an $m$-dimensional vector space, $n \leq m$, either generated by the same node or by a set of different nodes, are linearly independent. This can be achieved using, for instance, the well known random linear network coding scheme [4], and it ensures that the forwarded network coded packets are innovative with high probability.

\section{MODEL}

For simplicity, we first consider the (unrealistic) scenario of a lossless wireless mesh network. Consider the case with $|K|$ information flows. We denote with $s^{k}, d^{k}$ the source and destination node for commodity $k \in K$, or simply, $s, d$, when $k$ is clear from the context. To simplify the notation, we assume w.l.o.g. that source nodes, $\left\{s^{k}\right\}$, do not have incoming edges and destination nodes, $\left\{d^{k}\right\}$, do not have outgoing edges. If this is not the case, we can always consider a new graph obtained from $G$ by adding a super-source node connected to all and only the source nodes; similarly, we can connect all destination nodes to a single super-destination node without outgoing edges.

We assume that network coding operations happen only among packets of the same flow (intra-flow coding). We consider the scenario where no additional inter-session network coding is not used. ${ }^{1}$

Let $U(\cdot)$ be a differentiable, strictly concave, increasing function which represents the utility at every source node as a function of the sending rate. Finally, we indicate with $c$ the vector of capacities $\left\{c_{i j}\right\}$, and with $\Pi$ the set of feasible rates determined by the underlying physical layer used as described in [10], [11].

In this scenario, we can model the network utility maximization problem as follows:

$\mathcal{P}$ :

$$
\begin{aligned}
& \max _{z_{i}^{k} \geq 0, c_{i j} \geq 0} \sum_{k \in K} U\left(z_{s^{k}}^{k}\right) \\
\text { s.t. } \quad & \quad \forall k \in K \\
& \sum_{j>d^{k}} z_{j}^{k}=z_{s^{k}}^{k} \\
\sum_{j>i} z_{j}^{k} \geq z_{i}^{k} & \forall i \neq s^{k}, k \in K \\
\sum_{k \in K} z_{i}^{k} \leq c_{i j} & \forall i, \forall j: j<i \\
c & \in \Pi
\end{aligned}
$$

Problem $\mathcal{P}$ aims at maximizing the aggregate utility of sources $s^{k}$ as a function of, $z_{s^{k}}^{k}$ the number of packets generated. Note that given Eq.(2), this problem is equivalent to maximizing the number of packets received at the destination nodes. In the case of a lossless network with intra-session network coding, this also represents the number of linearly independent (l.i.) packets generated at the source node ${ }^{2}$. In other words, we can interpret $\mathcal{P}$ as maximizing the sum of the dimension of the vector spaces generated at source nodes, $\left\{s^{k}\right\}$. The higher the dimension of the vector space, the higher the utility of the corresponding source node.

Eq.(2)-(3) represent the mass conservation constraint in the frame of opportunistic routing with network coding. This is quite different from the traditional conservation equation used in different scenarios [10], [12]. In the case of opportunistic routing and network coding, no path is computed explicitly. Instead, multiple paths are opportunistically used taking advantage of the broadcast nature of the wireless transmissions. Then, Eq.(2) ensures that the number of 1.i. packets generated at source $s^{k}$ is equal to the number of 1.i. packets received at destination, $d^{k}$, so that the destination node is actually able to decode and recover the original information. Eq.(3) ensures that the dimension of the vector space spanned by outgoing packets cannot exceed the dimension of the vector space spanned by incoming packets, i.e. that intermediate nodes do

\footnotetext{
${ }^{1}$ In general, unless opportunistic decoding is immediately possible as in [6], [7], coding packets assigned to different destination nodes would cause each receiver to require additional information to decode the received coded packet. This is not always possible under capacity constraints and it depends on both the network topology and the traffic demands. The multiple unicast scenario with network coding is in general a difficult problem, not fully understood yet [8]. In random topologies, it has been observed through simulations that inter-session network coding does not bring significant throughput gain [9]. Finally, we observe that opportunistic inter-session network coding, (e.g. [6]), can easily be deployed on the top of intra-session network coding schemes.

${ }^{2}$ With a slight abuse of language, we refer to packets as linearly independent when the coding vectors associated to those packets are linearly independent.
} 


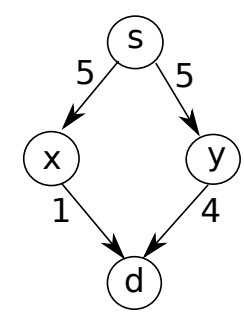

Fig. 1. An example of a diamond topology with 4 nodes. The labels on the edges indicate the number of packets received. Given the broadcast nature of the wireless channel, assuming no losses, both nodes $x$ and $y$ receive all 5 (1.i.) packets sent by source node $s$. In order to forward this information to destination node $d, x$ and $y$ do not need to send 5 packets each. Any solution such that $d$ receives precisely 5 (1.i.) packets will enable $d$ to decode the original information.

not generate new information. However, intermediate nodes can drop some (or all) of the received packets, for instance, as illustrated in Fig.1.

Finally, Eq.(4) imposes that enough capacity is allocated to support transmissions, and Eq.(5) ensures that they represent a feasible rate assignment, [10].

\section{A. Decomposition and Interpretation}

There exist different ways to solve $\mathcal{P}$. In the following, we apply a dual decomposition of $\mathcal{P}$, followed by a primal decomposition of the dual, and show how this leads to a simple and intuitive algorithm to solve $\mathcal{P}$. A tutorial paper with a detailed description of decomposition methods for network utility maximization can be found in [13]. Consider the (partial) dual of $\mathcal{P}$ obtained relaxing Eq.(2), Eq.(4):

$\mathcal{D}$ :

$$
\min _{p \geq 0, q} D(p, q)
$$

where, the dual function, $D$, is defined as:

$$
\begin{aligned}
\mathrm{D}(p, q)= & \max _{z_{i}^{k} \geq 0, c_{i j} \geq 0} \\
& \sum_{k \in K} U\left(z_{s^{k}}^{k}\right)-\sum_{i} \sum_{j<i} p_{i j}\left(\sum_{k \in K} z_{i}^{k}-c_{i j}\right)+ \\
& -\sum_{k \in K} q_{s^{k}}\left(z_{s^{k}}^{k}-\sum_{j>d^{k}} z_{j}^{k}\right) \\
\text { s.t. } & \sum_{j>i} z_{j}^{k} \geq z_{i}^{k} \quad \forall i \neq s^{k}, k \in K \\
& c \in \Pi
\end{aligned}
$$

We observe that while variable $p$ must be non-negative, $q$ is unconstrained. $D(p, q)$ decomposes into three subproblems, D1:

$$
\max _{z_{s^{k}}} \sum_{k \in K}\left(U\left(z_{s^{k}}^{k}\right)-\left(p_{s}+q_{s^{k}}\right) z_{s^{k}}\right)
$$

$\mathcal{D} 2$ :

$$
\begin{array}{ll}
\max _{z_{i} \geq 0, i \neq s} & \sum_{k \in K}\left(q_{s^{k}} \sum_{j>d^{k}} z_{j}^{k}-\sum_{i \neq s} \sum_{j<i} p_{i j} z_{i}^{k}\right) \\
\text { s.t. } \quad \sum_{j>i} z_{j}^{k} \geq z_{i}^{k} & \forall i \neq s
\end{array}
$$

D3:

$$
\begin{aligned}
& \max _{c \geq 0} \sum_{j<i} p_{i j} c_{i j} \\
& \text { s.t. } \quad c \in \Pi
\end{aligned}
$$

where $p_{i}=\sum_{j<i} p_{i j}$.

Problem (6) can be solved using a subgradient method. $g$ is a subgradient of a function $f$ (not necessarily differentiable or even convex) at $x_{0}$ if and only if $f(x)+g\left(x_{0}-x\right)$ is an affine under estimator of $f$ at $x_{0}$. A subgradient method is a simple iterative algorithm to minimize (or maximize) a convex function. Given a convex function $f(x)$ and the $t$-th iterate, $x^{(t)}$, the subgradient methods computes the $(t+1)$-th as:

$$
x^{(t+1)}=x^{(t)}-\alpha_{t} g\left(x^{(t)}\right)
$$

where, $g\left(x^{(t)}\right)$ is any subgradient of $f$ at $x^{(t)}$, and $\alpha_{t}>0$ is the $t$-th step size. Subgradient methods are extremely simple to implement and they are suitable to a large family of different problems (e.g., to minimize any convex function, regardless whether or not it is differentiable, both in the unconstrained and constrained scenario). In particular, subgradient methods are particularly well-suited for optimizing dual functions [14]. Let us consider Problem (6). Dual variables $q_{s^{k}},\left\{p_{i j}\right\}$ are associated to primal constraints Eq.(2), Eq. (4), respectively. Thus, a valid subgradient method to solve Problem (6) will update its variables as follows:

$$
\begin{aligned}
& p_{i j}(t+1)=\left[p_{i j}(t)+\alpha_{i j}\left(z_{i}(t)-c_{i j}(t)\right)\right]^{+} \\
& q_{s^{k}}(t+1)=q_{s^{k}}(t)+\beta_{s^{k}}\left(z_{s^{k}}^{k}(t)-\sum_{j>d^{k}} z_{j}(t)\right)
\end{aligned}
$$

where $z_{i}(t), c_{i j}(t)$ are the values of the optimal solution of the subproblems $\mathcal{D} 1, \mathcal{D} 2, \mathcal{D} 3$, when $p_{i j}=p_{i j}(t), q_{s^{k}}=$ $q_{s^{k}}(t),[\cdot]^{+}$is the projection on the non-negative values, $[x]^{+}=x$ iff $x \geq 0,0$ otherwise, and, $\alpha_{i j}, \beta_{s^{k}}$ represent the step sizes associated with $p_{i j}$ and $q_{s^{k}}$ respectively

Let us now examine the structure and interpretation of the subproblems.

Problem $\mathcal{D} 1$ corresponds to congestion control. $\mathcal{D} 1$ has a similar structure to the TCP optimization problem [10], [15], [16] and has a single solution, $z_{s^{k}}^{k}=U^{\prime-1}\left(p_{s^{k}}+q_{s^{k}}\right)$. However, interestingly enough, there is an additional term, $q_{s^{k}}$. In fact, in previous approaches the authors assume the routing to be fixed (at the time scale considered). As a result, the reaction of the TCP source only depends on the price $p_{s k}$ which is interpreted as a measure of congestion (e.g. queueing delay). Here, we do not assume a fixed route from $s^{k}$ to $d^{k}$. Routes to $d^{k}$ are opportunistically selected in a hop-by-hop base, only upon successful packet receptions. This results in an additional price, $q_{s^{k}}$. We can interpret $q_{s^{k}}$ through Eq.(17). Every time the vector space generated at $s^{k}, z_{s^{k}}^{k}$, has higher dimension than the vector space received at destination, $\sum_{j>d^{k}} z_{j}$, which means that not all information generated at $s^{k}$ is successfully delivered to $d^{k}$, the price $q_{s^{k}}$ is increased. This, in return, increases the penalty (congestion) term in 
Eq.(11) on the sender side, and consequently reduces the sending rate. At an intermediate forwarding node, Eq.(12), it increases the sending rate of the neighbors of the $d$. Through Eq.(13), this increase propagates backward toward the source to increase the sending rate of all intermediate nodes involved in the forwarding process.

Thus, we can interpret $q_{s}$ as an end-to-end feedback that can be used to adjust the sending rate of the source based on what is actually received by the destination. The presence of this term is due to the fact that we do not have a fixed routing, where each intermediate node receives and forwards the same amount of information. Here, in order to reduce the overall number of transmissions needed, when the same information is successfully received by multiple nodes only one of them will trigger a transmission (for instance, the closer to $d^{k}$ in the ETX metric). The other nodes can safely store the packet, if any space is available, and use it for future coding operations; or discard it, if no more space is available at the node's buffer.

$\mathcal{D} 2$ represents the routing problem. It finds the routes that satisfy the conservation constraint Eq.(13) and have minimum total cost.

We note that $z_{i}=0 \forall i \neq s$ is a feasible solution of Eq.(12)(13). However, in this case $q_{s^{k}}$ will increase, according to Eq.(17), and thus cause an increase of the packets forwarded at intermediate nodes, $z_{i}$, through Eq.(12)-(13).

An alternative interpretation of Problem $\mathcal{D} 2$ is obtained by noting that Eq.(12)-(13) can equivalently be written as:

$$
\begin{aligned}
& \min _{z_{i} \geq 0, i \neq s} \sum_{k \in K}\left(\sum_{i \neq s} p_{i} z_{i}^{k}-\sum_{j>d^{k}} q_{s^{k}} z_{j}^{k}\right) \\
& \text { s.t. } \quad \sum_{j>i} z_{j} \geq z_{i}
\end{aligned}
$$

This can be interpreted as a generalization of the wellstudied job scheduling problem [17], [18]. For every commodity, $k$, we can think of variables $z_{i}^{k}$ as indicating the amount of time associated to job $i$. Every job has a cost (either positive or negative) assigned to it, according to the coefficient associate to $z_{i}^{k}$ in Eq.(18). Jobs must be performed sequentially according to the underlying connectivity graph $G$ : a new job can start only when at least one of the upstream jobs has been performed. Moreover, the amount of time assigned to job cannot exceed the amount of time assigned to predecessor (upstream) jobs, Eq.(19). Given these constraints the goal is to maximize the overall profit, Eq.(18).

Finally, $\mathcal{D} 3$ represents the wireless interference problem similarly to [12]. This problem well-known to be NP-Hard. Several heuristics have been proposed to achieve an approximate solution [10], [12], [19].

In summary, problem $\mathcal{P}$ is the network utility maximization problem in the presence of intra-session network coding and opportunistic routing to forward coded packets. Using a partial dual decomposition, and a primal decomposition on the dual problem, $\mathcal{P}$ decomposes into three subproblems, which are optimized at three different layers, namely, transport, routing, and MAC layers. The MAC only depends on congestion prices, while the transport and the routing depend also on an additional price which accounts for any discrepancy between the information sent at $s^{k}$ and the information received at $d^{k}$.

\section{B. Lossy links}

Let us denote with $\sigma_{i j}$ the loss probability over the logical link $(i, j)$. We assume $\sigma_{i j}$ to be constant over the time scale considered (possibly variable in longer time window). We also assume that reception at different nodes are independent, as reported in recent measurement studies [20].

In this scenario, problem $\mathcal{P}$ can be extended to model lossy networks as follows:

$$
\begin{aligned}
\mathcal{P}^{\prime}: & \\
\max _{z_{i}^{k} \geq 0, c_{i j} \geq 0} & \sum_{k \in K} U\left(z_{s}^{k}\right) \\
\text { s.t. } & \sum_{j>d} z_{j}^{k} \sigma_{j d}=z_{s}^{k}\left(1-\prod_{j<s} \sigma_{s j}\right) \quad \forall k \\
& \sum_{j>i} z_{j}^{k} \sigma_{j i} \geq z_{i}^{k}\left(1-\prod_{j<i} \sigma_{i j}\right) \quad \forall k, i \neq s \\
& \sum_{k \in K} z_{i}^{k} \sigma_{i j} \leq c_{i j} \quad \forall i, \forall j: j<i \\
& c \in \Pi
\end{aligned}
$$

Problem $\mathcal{P}$ ' again aims at maximizing the aggregate network utility. In the lossy case the number of packets successfully forwarded to the source's neighbors, $z_{s^{k}}^{k}\left(1-\prod_{j<s} \sigma_{s j}\right)$, is smaller than the number of packets generate at the source, $z_{s^{k}}^{k}$. To compensate for the random losses over the wireless medium, the source forwards a certain amount of redundant packets in addition to the innovative packets it generated. In particular, we have that $z_{s}^{k}=z_{s^{k}}^{\prime} R$, where $R=\frac{1}{1-\prod_{j<s} \sigma_{s j}}$ is the redundancy rate and $z_{s^{k}}^{\prime}$ is the number of innovative packets transmitted. Eq.(21) model the constraint that the aggregate amount of information sent from the source and successfully received by any neighbor node must be equal to the amount of information received by the destination node. Similarly to Eq.(3), Eq.(22) ensures that the vector space associated with outgoing packets does not exceed the vector space of incoming packets. In fact, a node can receive a packet and decide not to forward it (e.g. another neighboring node is in charge of that transmission). However, an intermediate node, by definition, cannot generate new information.

Finally, we note that, there is a side benefit in this optimization model: estimating the loss rate probability does not require additional overhead, because opportunistic routing schemes, [2], [1], estimate and propagate those quantities anyway in order to compute the best routes.

In order to solve $\mathcal{P}^{\prime}$, we can consider its dual problem:

$$
\min _{p \geq 0, q} D(p, q)
$$


where:

$$
\begin{aligned}
& D(p, q)= \max _{z \geq 0, c \geq 0} \sum_{k \in K}\left[U\left(z_{s}^{k}\right)-p_{i j}\left(z_{i}^{k} \sigma_{i j}-c_{i j}\right)+\right. \\
&\left.-\sum_{k \in K} q_{s}\left(\sum_{j>d} z_{j}^{k} \sigma_{j d}-z_{s}^{k}\left(1-\prod_{j<s} \sigma_{s j}\right)\right)\right] \\
& \sum_{j>i} z_{j}^{k} \sigma_{j i} \geq z_{i}^{k}\left(1-\prod_{j<i} \sigma_{i j}\right) \quad \forall i \neq s \\
& c \in \Pi
\end{aligned}
$$
$\mathcal{P}$.

and apply the same methodology as in the case of Problem

\section{Insight Into PRotocol DESIGN}

In the previous section, we have presented a simple optimization model that accounts for the interaction between the transport layer and opportunistic routing, network coding and the wireless medium. We have also developed a distributed solution that has an intuitive interpretation. In this section, we discuss how the insight gained from the analysis can be used for protocol design.

The opportunistic routing constraints are associated with the dual variables, $\left\{q_{s^{k}}\right\}$, which is explicitly tied to the difference between the dimension of vector space generated by the source and the dimension of the vector space received at destination, Eq.(17). Contrary to prior work (such as [10], which defined the congestion prices as local variables), $q_{s^{k}}$ explicitly models an intuitive end-to-end signaling: source $s^{k}$ adjust its sending rate based on the price $q_{s}^{k}$, which in turn depends on both the current sending rate of $s^{k}$ and the information received at destination node $d^{k}$ trough Eq.(17). The importance of fast end-to-end signaling is crucial for adjusting the source sending rate. For this same reason, the online network coding approach in [3] is more suitable for TCP than generation-based schemes such as the one in [4].

Problem $\mathcal{D} 2$ gives us further insights. Consider the relaxation of $\mathcal{D} 2$ with respect to constraint Eq.(13),

$\max _{z_{i} \geq 0, i \neq s} \sum_{k \in K}\left(q_{s^{k}} \sum_{j>d^{k}} z_{j}^{k}-\sum_{i \neq s} p_{i} z_{i}^{k}-\sum_{i \neq s} q_{i}\left(z_{i}^{k}-\sum_{j>i} z_{j}^{k}\right)\right)$

this can be rewritten as,

$$
\begin{gathered}
\max _{z_{i} \geq 0, i \neq s} \sum_{k \in K}\left(\sum_{\substack{i \neq s \\
i \notin\left\{j>d^{k}\right\}}} z_{i}^{k}\left(q_{\text {out }(i)}-q_{i}-p_{i}\right)+\right. \\
+\sum_{\substack{i \neq s \\
i \notin\left\{j>d^{k}\right\}}} z_{i}^{k}\left(q_{s}+q_{\text {out }(i)}-q_{i}-p_{i}\right)
\end{gathered}
$$

where $q_{\text {out }(i)}$ represents the sum of prices $q_{j}$ associated with downstream nodes of node $i, q_{\text {out }(i)}=\sum_{j<i} q_{j}$.

We can see that the coefficient of variables $\left\{z_{i}\right\}$ depends on the (local) congestion prices $p_{i j}$ and on the difference $\left(q_{\text {out }(i)}-q_{i}\right)$. This can be interpreted as the back-pressure at node $i$ generated by the union of its downstream neighbors.
A node which has more information than its downstream neighbors will be selected for transmission. However, as this difference becomes close to zero, so does the sending rate of $i$. This has an intuitive explanation since in the case of network coding and opportunistic routing we do not force a precise route. Instead, we just require that the vector space transmitted by vectors at $i$ is received by any of the downstream nodes.

\section{RELATED WORK}

Since the seminal paper [21], network utility maximization models have been long studied and generalized to different types of networks (see [22], [23] and references therein). This work falls within the mathematical framework of "layering as optimization decomposition" [22]. However, compared to prior works, [12], [10], [24], we consider a different scenario where both opportunistic routing and network coding are used. Opportunistic routing for wireless mesh was introduced and experimentally tested in [2], which showed large benefits over traditional routing. The main idea behind [2] is to choose the next hop of a packet's route only after the packet has been successfully received by the sender's neighbors. In this way, a packet opportunistically leverages nodes' reception as they happen to approach its destination as fast as possible. In [1], authors exploits the use of generation-based network coding to further improve the performance [2]. A sliding-window network coding scheme was introduced in [25], [3].

The most closely related work to ours is [19] and its extended version [5]. We consider the same problem as in [5]. However, our work differs both in the formulation (control variables) and in the decomposition methods used. In fact, we design our model considering the vector space spanned by the coding vectors at a each node, while in [5] the authors consider a system of credits transferred between logical edges between nodes. This results in a different and more intuitive model for the conservation equations. Finally, the solution of our model is obtained in a simpler way, applying a sub-gradient method to the dual problem.

\section{CONCLUSION}

Opportunistic routing deals with the unpredictable losses of the wireless medium by allowing all nodes that successfully received the transmission to coordinate to forward it to the destination. This approach was proven to be beneficial in real implementations of wireless mesh networks [2]. However, it requires additional coordination among nodes. This overhead can be greatly reduced with network coding by not forwarding a single packet but a combination of them [1].

In this work, we consider MORE [1] and we model the joint optimization of congestion control, opportunistic routing and network coding in wireless mesh networks as a network utility maximization problem. Dual algorithms have found an extensive number of applications both in wired and wireless networks [22]. We apply and extend this framework to the considered scenario, and we show how it immediately leads to an algorithm that is both simple to implement and to interpret. 
This provides a formal foundation and useful insights in the fundamental design of real protocols.

This paper focuses on the optimization model, solution and interpretation. Directions for future work include building a system, based on the intuition gained, and evaluating the benefits from this optimization in practice.

\section{REFERENCES}

[1] S. Chachulski, M. Jennings, S. Katti, and D. Katabi, "Trading structure for randomness in wireless opportunistic routing," SIGCOMM Comput. Commun. Rev., vol. 37, no. 4, pp. 169-180, 2007.

[2] S. Biswas and R. Morris, "Opportunistic routing in multi-hop wireless networks," SIGCOMM Comput. Commun. Rev., vol. 34, no. 1, pp. 69 74, 2004.

[3] J. K. Sundararajan, D. Shah, M. Medard, M. Mitzenmacher, and J. Barros, "Network coding meets tcp," in in Proceedings of IEEE INFOCOM, 2008. [Online]. Available: http://www.citebase.org/abstract? id=oai:arXiv.org:0809.5022

[4] T. Ho, M. Medard, J. Shi, M. Effros, and D. R. Karger, "On randomized network coding," in In Proceedings of 41st Annual Allerton Conference on Communication, Control, and Computing, 2003.

[5] B. Radunovic, C. Gkantsidis, P. Key, and P. Rodriguez, "Toward practical opportunistic routing with intra-session network coding for mesh networks," in in Proceedings of IEEE Transaction on Networking, 2009.

[6] S. Katti, H. Rahul, D. Katabi, M. Medard, and J. Crowcroft, "XORs in the Air: Practical Wireless Network Coding," IEEE/ACM Transactions on Networking, vol. 16, no. 3, pp. 497-510, June 2008 [Online]. Available: http://ieeexplore.ieee.org/lpdocs/epic03/wrapper htm?arnumber $=4512941$

[7] S. Omiwade, R. Zheng, and C. Hua, "Butteries in the Mesh Lightweight Localized Wireless Network Coding," Fourth Workshop on Network Coding, Theory and Applications, vol. 2, pp. 1-6, January 2008. [Online]. Available: http://ieeexplore.ieee.org/lpdocs/ epic03/wrapper.htm?arnumber $=4476174$

[8] N. Harvey, R. Kleinberg, and A. Lehman, "On the capacity of information networks," IEEE Transactions on Information Theory, vol. 52, no. 6, pp. 2345-2364, June 2006. [Online]. Available: http: //ieeexplore.ieee.org/lpdocs/epic03/wrapper.htm?arnumber=1638531

[9] D. Traskov, N. Ratnakar, D. S. Lun, and R. Koetter, "Network Coding for Multiple Unicasts: An Approach based on Linear Optimization,' ISIT, no. v, 2006.

[10] L. Chen, S. H. Low, M. Chiang, and J. C. Doyle, "Cross-layer congestion control, routing and scheduling design in ad hoc wireless networks," in INFOCOM 2006. 25th IEEE International Conference on Computer Communications. Proceedings, 2006, pp. 1-13. [Online]. Available: http://dx.doi.org/10.1109/INFOCOM.2006.142

[11] K. Jain, J. Padhye, V. N. Padmanabhan, and L. Qiu, "Impact of interference on multi-hop wireless network performance," in MobiCom '03: Proceedings of the 9th annual international conference on Mobile computing and networking. New York, NY, USA: ACM, 2003, pp. 66-80.

[12] L. Chen, S. H. Low, and J. C. Doyle, "Joint congestion control and media access control design for ad hoc wireless networks," in in Proceedings of IEEE INFOCOM, 2005, pp. 2212-2222.

[13] D. Palomar and M. Chiang, "A tutorial on decomposition methods for network utility maximization," Journal on Selected Areas in Communications, vol. 24, no. 8, pp. 1439-1451, August 2006. [Online]. Available: http://ieeexplore.ieee.org/lpdocs/epic03/wrapper. htm?arnumber $=1664999$

[14] S. Boyd, "Convex optimization 2, lecture notes." [Online]. Available: http://www.stanford.edu/class/ee364b/lectures.html

[15] F. Kelly, A. Maulloo, and D. Tan, "Rate control in communication networks: shadow prices, proportional fairness and stability," in Journal of the Operational Research Society, vol. 49, 1998. [Online]. Available: citeseer.ist.psu.edu/kelly98rate.html

[16] S. H. Low, "A duality model of tcp and queue management algorithms," IEEE/ACM Trans. on Networking, vol. 11, pp. 525-536, 2002.

[17] M. Brusco and L. Jacobs, "Cost analysis of alternative formulations for personnel scheduling in continuously operating organizations," European Journal of Operational Research, vol. 86, no. 2, pp. 249-261, October 1995. [Online]. Available: http://linkinghub.elsevier. com/retrieve/pii/037722179400063I
[18] P. Brucker, Scheduling algorithms. Springer Verlag, 2007.

[19] B. Radunovic, C. Gkantsidis, P. Key, and P. Rodriguez, "An optimization framework for opportunistic multipath routing in wireless mesh networks," in in Proceedings of IEEE mini INFOCOM, 2008.

[20] C. Reis, R. Mahajan, M. Rodrig, D. Wetherall, and J. Zahorjan, "Measurement-based models of delivery and interference in static wireless networks," SIGCOMM Comput. Commun. Rev., vol. 36, no. 4, pp. 51-62, 2006.

[21] F. Kelly, A. Maulloo, and D. Tan, "Rate control in communication networks: shadow prices, proportional fairness and stability," in Journal of the Operational Research Society, vol. 49, 1998. [Online]. Available: citeseer.ist.psu.edu/kelly98rate.html

[22] M. Chiang, S. H. Low, A. R. Calderbank, and J. C. Doyle, "Layering as optimization decomposition: A mathematical theory of network architectures," in Proceedings of the IEEE, 2007.

[23] — "Layering as optimization decomposition: Questions and answers," IEEE MILCOM, 2006.

[24] B. Radunovic, C. Gkantsidis, D. Gunawardena, and P. Key, "Horizon: Balancing tcp over multiple paths in wireless mesh network," in in Proceedings of ACM Mobicom, 2009.

[25] J. K. Sundararajan, D. Shah, and M. Medard, "ARQ for network coding," IEEE Information Theory, ISIT, pp. 1651-1655, July 2008. [Online]. Available: http://ieeexplore.ieee.org/lpdocs/epic03/ wrapper.htm?arnumber $=4595268$ 